Check for updates

Cite this: RSC Adv., 2017, 7, 49863

Received 27th July 2017

Accepted 10th October 2017

DOI: 10.1039/c7ra08318a

rsc.li/rsc-advances

\section{Synthesis, structure-property and flame retardancy relationships of polyphosphonamide and its application on epoxy resins $\uparrow$}

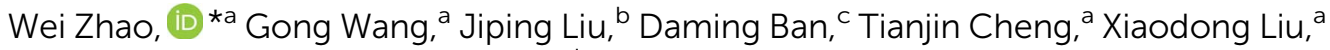 \\ Yongxiang $\mathrm{Li}^{\mathrm{a}}$ and Yongqing Huang ${ }^{\mathrm{d}}$
}

A series of polyphosphonamides (PPDA) with heteroatoms and phosphonamide structures are proposed as highly effective flame retardants in epoxy resins (EP). The PPDAs were synthesized by solution polycondensation and well characterized. The impact of the heteroatoms on the thermal properties, pyrolysis route and flammability of PPDAs and their EP composites was investigated in detail. All the PPDAs show good thermal stability, high glass transition temperatures $\left(T_{\mathrm{g}}\right)$ and low flammability, associated to their backbone structures. With the addition of PPDAs, the flame retardancy of the composites was gradually enhanced. It is noteworthy that the heat release rate and thermal properties highly depend on the chemical environment of the phosphorus atom in PPDAs. The flame retardant mechanisms are suggested to enhance the charring of EP, protect the matrix in the condensed phase, and have a flame inhibition effect in the gas phase. The potential for increasing flame retardancy and maintaining high $T_{\mathrm{g}}$ and high fracture toughness is highlighted in this study.

\section{Introduction}

With the rapid development in the design of lightweight materials and advanced composites in modern society, epoxy resins (EPs) have found increasing use as engineering adhesives and as the matrix of fiber reinforced polymers in applications such as new energy automobiles and complex structural aircraft components..$^{1-4}$ For their application in these thriving markets, high-performance EPs, with good physical properties, high material compatibility and high flame retardancy, need to be developed and fabricated. ${ }^{5,6}$ EP burns at a rapid heat release rate, producing small amounts of chars, and is unable to achieve any UL-94 rating. ${ }^{7}$ To obtain a higher flame retardant class, EP requires the addition of chemicals characterized by higher flame retardancy. Traditional EP are mainly prepared by blending with additional compounding with flame retardant additives. ${ }^{8-11}$ However, a major problem encountered in this system is that the high loading (20 wt\%) leads to deterioration of physical properties of EP. ${ }^{\mathbf{1 2 - 1 4}}$

${ }^{a}$ Technology and Engineering Center for Space Utilization, Chinese Academy of Sciences, Beijing, 100094, China. E-mail: zhaowei@csu.ac.cn

${ }^{b}$ School of Material Science and Engineering, Beijing Institute of Technology, Beijing, 100081, China

'School of Chemistry and Material Science, Guizhou Normal University, Guiyang, 550001, China

${ }^{d}$ College of Chemical and Environmental Engineering, Shandong University of Science and Technology, Qingdao, 266590, China

$\dagger$ Electronic supplementary information (ESI) available. See DOI: $10.1039 / \mathrm{c} 7 \mathrm{ra0} 3318 \mathrm{a}$
Researchers have attempted many methods to develop novel flame retardant for $\mathrm{EP}$, such as layered double hydroxide $(\mathrm{LDH}),{ }^{15-17}$ graphene, ${ }^{18,19}$ and phenylphosphonicdiamide. ${ }^{19-21}$

Polymeric flame retardants have shown the advantages of low toxicity, good compatibility, and negligible negative impact on polymer physical properties. ${ }^{22,23}$ Schartel et al. synthesized a phosphorus-modified polysulfone as both flame retardant and toughness modifier for epoxy resins, and proved that polyphosphates with a hyperbranched structure were more efficient than low-molecular-weight bisphenol A bis(diphenylphosphate) (BDP). ${ }^{\mathbf{2 4 5}}$ Wang et al. reported that $\mathrm{EP} /$ polyphosphate composites exhibit a higher glass transition temperature than pure EP..$^{\mathbf{2 6} 27} \mathrm{Up}$ to now, polymeric flame retardants were mostly represented by polyphosphates (PPEs); however, high loadings were always required to meet the desired flame retardant grade because of their poor flame retardancy. ${ }^{28,29}$

As families of polymeric flame retardant, polyphosphonamide (PPDAs) have been only scarcely investigated to date. In addition to nitrogen, PPDAs have shown a synergistic effect of $\mathrm{P}$ and $\mathrm{N}$ to give high thermal stabilities and high flame retardancy. ${ }^{30-32}$ Tai et al. prepared PPDAs with high char residues, relatively high molecular weight and low flammability. ${ }^{33}$ Steinmann et al. prepared a series of PPDAs and PPEs with pendant $\mathrm{P}-\mathrm{O}-\mathrm{C}$ structure by acyclic diene metathesis polycondensation, and the obtained PPDAs showed higher thermal stability and higher glass transition temperatures than the analogous PPEs. ${ }^{34}$ Li et al. prepared a hyperbranched PPDAs, and found that the flame retardant PLA achieved UL-94 
V-0 rating with only $2 \mathrm{wt} \%$ the hyperbranched PPDAs. ${ }^{35}$ Such PPDAs possessed impressive high thermal stability and high flame retardancy, but their pendant $\mathrm{P}-\mathrm{O}-\mathrm{C}$ structure often tend to hydrolyze under basic conditions. ${ }^{36,37}$ Of note, fewer researchers reported that $\mathrm{P}-\mathrm{C}$ structure possesses excellent hydrolysis stability and higher flame retardancy than $\mathrm{P}-\mathrm{O}-\mathrm{C}$ structure, and such reports hint at potential development of PPDAs with high flame retardancy. ${ }^{\mathbf{3 8 - 4 0}}$ Zhao et al. systemically studied the impact of two types of reactive flame retardant (FR) with $\mathrm{P}-\mathrm{C}$ and $\mathrm{P}-\mathrm{O}-\mathrm{C}$ structure on the flame retardant efficiency of EP (DGEBA/DDS system). ${ }^{41}$ The FR with P-C structure showed flame inhibition effect in the gas phase and endow EP with strong self-extinguishing ability at a low phosphorus content $(0.7 \mathrm{wt} \%)$, whereas FR with $\mathrm{P}-\mathrm{O}-\mathrm{C}$ structure failed to achieve any rating. PPDAs with pendant $\mathrm{P}-\mathrm{C}$ structure via polycondensation is plausible, although, to the best of our knowledge, such PPDAs have never been applied to develop flame retardant EP.

In this work, we prepared a series of PPDAs with pendent $\mathrm{P}-\mathrm{C}$ structure by solution polycondensation of phenylphosphonic dichloride and aromatic diamines. The PPDAs have been characterized by ${ }^{1} \mathrm{H}$ NMR and ${ }^{31} \mathrm{P}$ NMR spectroscopy, and applied into flame retardant EPs. To prove the structure-property relationships and widely-useful of the phosphonamide structure with pendent $\mathrm{P}-\mathrm{C}$ structure, aromatic diamines with methylene group, sulfone group and ether group were employed as staring materials. The thermal stability and flame retardancy of the synthesized PPDAs and flame retardant EP were investigated. The thermal properties of PPDAs and flame retardant EP, such as glass transition temperatures, highly depend on the introduction of microstructure. Besides, the impact of PPDAs on the EP/PPDA composites was studied. More importantly, the structure-property relationships in flame retardant EP were investigated in detail.

\section{Experimental}

\subsection{Materials}

Phenylphosphonic dichloride was purchased from SigmaAldrich. 4,4'-Diaminodiphenyl sulfone, 4,4'-diaminodiphenyl methane, 4,4'-diaminodiphenyl ether, $m$-phenylenediamine (m-PDA), dimethyl sulfoxide (DMSO) and ethanol were purchased from Sinopharm Chemical Reagent Beijing Co., Ltd
(Beijing, China), were all reagent grade and used as received. Acetonitrile was purchased from Tianjin Guangfu Fine Chemical Research Institute (Tianjin, China) and freshly distilled before use. Epoxy resin (DGEBA, commercial name: E-44) was supplied by Sinopec Baling Company (Yueyang, China).

\subsection{Synthesis of PPDAs}

A typical synthesis of poly $\left(4,4^{\prime}\right.$-diamino diphenyl sulfone phenyl phosphonamide) (POS) is given below.

In a dry $250 \mathrm{~mL}$ three-neck and round-bottom glass flask equipped with a constant pressure funnel, mechanical stirrer and a reflux condenser, $24.8 \mathrm{~g}(0.1 \mathrm{~mol})$ of 4,4'-diaminodiphenyl sulfone and $80 \mathrm{~mL}$ acetonitrile were mixed at $60{ }^{\circ} \mathrm{C}$ for $1 \mathrm{~h}$. Then, a solution of $19.5 \mathrm{~g}(0.1 \mathrm{~mol})$ phenylphosphonic dichloride in $20 \mathrm{~mL}$ acetonitrile was added dropwise to the mixture over $1 \mathrm{~h}$. The temperature of the reaction mixture was gradually raised to refluxing temperature and maintained for another $12 \mathrm{~h}$ under a nitrogen gas atmosphere. After cooling to room temperature, the precipitate was filtered and washed with acetonitrile. The crude mixture was then dissolved in minimum of DMSO, and then precipitated into to ethanol give fluffy grey white solid powders which were washed with ethanol. The product was then dried under reduced pressure to give a grey white powder $(87 \%)$. All the reaction processes are shown in scheme 1.

The other two PPDAs, poly $\left(4,4^{\prime}\right.$-diamino diphenyl methane phenyl phosphonamide) (POM) and poly $\left(4,4^{\prime}\right.$-diamino diphenyl ether phenyl phosphonamide) (POA) were also prepared in the described method above.

\subsection{Preparation of EP/PPDA composites}

The obtained PPDAs were used as additive-type flame retardants to combine with DGEBA at $80{ }^{\circ} \mathrm{C}$ and mixed in a IKA RW 20 digital stirrer for $10 \mathrm{~min}$, respectively. Then, the curing agent $m$-PDA were added and mixed with the same method for $3 \mathrm{~min}$. The formulation was poured into an PTFE mould and cured at $80{ }^{\circ} \mathrm{C}$ for $2 \mathrm{~h}$ and post-cured at $120^{\circ} \mathrm{C}$ for $2 \mathrm{~h}$. All samples were slowly cooled to room temperature to avoid stress cracking. In order to determine the flame retardancy of the PPDAs, all samples were prepared with constant loadings of $15 \mathrm{wt} \%$ of PPDA, respectively.
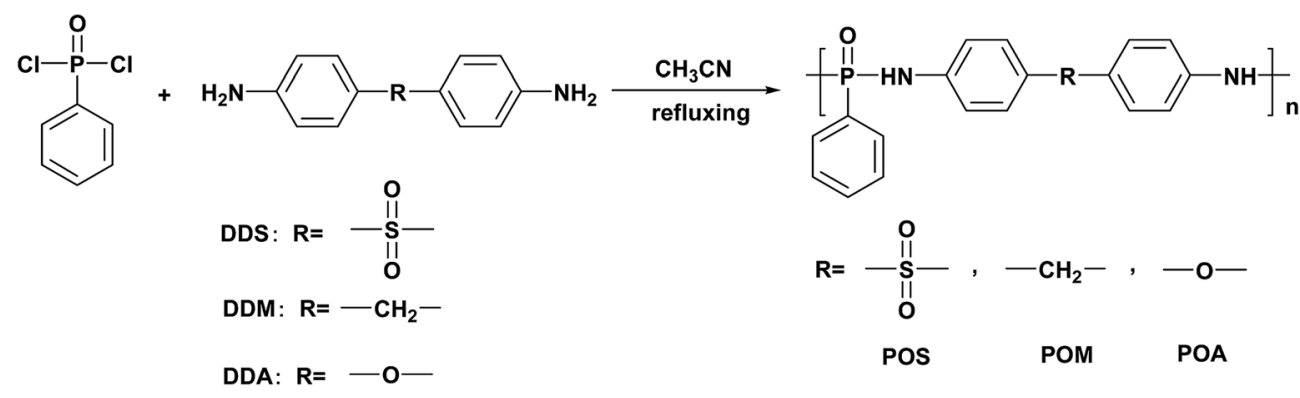

Scheme 1 Synthesis route of POS, POM and POA. 


\subsection{Measurements and characterization}

2.4.1 Fourier transform infrared spectroscopy. FTIR spectra were obtained over the range 4000-400 $\mathrm{cm}^{-1}$ on a Bruker Tensor 27 Fourier transform spectrophotometer from samples in $\mathrm{KBr}$ pellets.

2.4.2 Nuclear magnetic resonance spectroscopy. ${ }^{1} \mathrm{H}$ NMR (400 MHz) and ${ }^{31} \mathrm{P}$ NMR (163 MHz) were recorded on a Bruker AVANCE NMR spectrometer. All ${ }^{1} \mathrm{H}$ NMR and ${ }^{31} \mathrm{P}$ NMR were obtained in $d_{6}$-DMSO and referenced to residual protonated solvent and phosphoric acid, respectively.

2.4.3 Gel permeation chromatography. GPC was performed on a Waters Breeze ${ }^{\mathrm{TM}} 2$ HPLC system with a 2489 UV/Visible detector and a 1515 isocratic HPLC pump. The PPDAs were eluted with DMF at a flow rate of $0.5 \mathrm{~mL} \mathrm{~min}^{-1}$. Both the column and the detector were maintained at $40{ }^{\circ} \mathrm{C}$ during the determination process.

2.4.4 Differential scanning calorimetry. The DSC curves of the PPDAs and prepared EP/PPDA composites were carried out with a DSC Q2000 (TA Ltd., USA). About $5 \mathrm{mg}$ of sample were put in a aluminum crucible and tested at a heating rate of $20{ }^{\circ} \mathrm{C} \min ^{-1}$ under nitrogen. The glass transition temperature $\left(T_{\mathrm{g}}\right)$ was read at the mid-point of the inflection curve from the typical second heating in the range of $40-220{ }^{\circ} \mathrm{C}$.

2.4.5 Thermalgravimetric analysis/Fourier-transform infrared spectroscopy (TGA-FTIR). TGA was performed on a Mettler-Toledo TGA/DSC-1 thermalgravimetric analyzer under nitrogen atmosphere. Approximately $5 \mathrm{mg}$ of the samples were weighted in alumina crucible and heated from 50 to $700{ }^{\circ} \mathrm{C}$ at a rate of $20{ }^{\circ} \mathrm{C} \mathrm{min}^{-1}$. The FTIR information of the pyrolysis gases of the samples were obtained by Bruker Tensor 27 Fourier transform spectrophotometer coupled with TGA. The FTIR analysis was performed with a resolution of $4 \mathrm{~cm}^{-1}$ ranging from 4000 to $550 \mathrm{~cm}^{-1}$. The thermogravimetric analyzer and FTIR spectrometer were connected by a quartz capillary at $300{ }^{\circ} \mathrm{C}$.

2.4.6 Pyrolysis combustion flow calorimetry (PCFC). The flammability characteristics of the PPDAs were conducted on a Govmark MCC-2 microscale combustion calorimeter according to ASTM D 7309. Typical $5 \mathrm{mg}$ of samples were heated from 50 to $700{ }^{\circ} \mathrm{C}$ at $1 \mathrm{~K} \mathrm{~s}^{-1}$ in a inert gas steam $\left(80 \mathrm{~mL} \mathrm{~min}{ }^{-1}\right)$. The pyrolysis products were then mixed with oxygen $\left(20 \mathrm{~mL} \mathrm{~min}^{-1}\right)$ prior to entering a $900{ }^{\circ} \mathrm{C}$ combustion furnace. The heats release of combustion of the pyrolysis products were calculated by the oxygen consumption principle.

2.4.7 Cone calorimeter test. Flame retardant behavior was carried out on a Fire Testing Technology apparatus at a heat flux of $50 \mathrm{~kW} \mathrm{~m} \mathrm{~m}^{-2}$ according to ISO $5660-1$, and the size of the specimens was $100 \times 100 \times 1.2 \mathrm{~mm}^{3}$. All samples were mounted on aluminum foil and tested for three times, and the data were averaged.

2.4.8 Limit oxygen index (LOI) measurement. The LOI value was measured on a JF-3 oxygen index meter (Nanjing Jiangning Analysis Instrument Co., China) according to ASTM D2863. The specimens were prepared with dimensions of $130 \times$ $6.5 \times 3 \mathrm{~mm}^{3}$ by molding.

2.4.9 Vertical burning test. The vertical burning test were determined on a CZF-3 instrument (Nanjing Jiangning Analysis
Instrument Co., China) with dimensions of $125 \times 12.7 \times$ $3.2 \mathrm{~mm}^{3}$ according to ASTM D3801.

2.4.10 X-Ray photoelectron spectroscopy (XPS). The XPS data were obtained from a PHI Quantera II SXM at $25 \mathrm{~W}$ under a vacuum lower than $10^{-6} \mathrm{~Pa}$.

2.4.11 SEM characterization. The morphologies of char residue obtained from Cone tests were observed by a Hitachi S4800 scanning electron microscope with a conductive gold layer.

2.4.12 Fracture toughness. The fracture toughness $\left(K_{\mathrm{IC}}\right)$ of the prepared epoxy resins was measured from the opening mode test according to ASTM D 5045, performed on a MTS 810 material testing machine (USA). The size of the specimens used in the study was $50 \times 48 \times 5 \mathrm{~mm}^{3}$. A razor blade was used to generate the pre-crack. At least four specimens of each formulation were tested at room temperature.

2.4.13 Pyrolysis-GC/MS. The pyrolyses were conducted on a EGA/PY-3030D (Frontier Laboratories, Japan) attached to a Agilent 6890N GC/MS system (Agilent Technologies Inc., USA). About $1 \mathrm{mg}$ of samples was pyrolyzed at $500{ }^{\circ} \mathrm{C}$ for $1 \mathrm{~min}$ in a quartz tube. The temperature of the GC injector was $280{ }^{\circ} \mathrm{C}$. The oven temperature was held at $50{ }^{\circ} \mathrm{C}$ for $5 \mathrm{~min}$, then increased to $270{ }^{\circ} \mathrm{C}$ by applying a ramp of $30{ }^{\circ} \mathrm{C} \mathrm{min}$. The MS conditions were as follows: electron ionization mode, ionization energy $70 \mathrm{eV}$, ion source temperature $220{ }^{\circ} \mathrm{C}, \mathrm{GC} / \mathrm{MS}$ transfer line temperature $270^{\circ} \mathrm{C}$, and full-scan mode $\mathrm{m} / \mathrm{z} 30-400,1$ scan per $\mathrm{s}$ with a dwell time of $0.2 \mathrm{~s}$.

\section{Results and discussion}

\subsection{Synthesis and characterization of PPDAs}

3.1.1 Structural characterization. The PPDAs were synthesized through a direct solution polycondensation of phenylphosphonic dichloride and aromatic diamines (Scheme 1). For PPDAs, typically acid-binding agents, such as triethylamine, pyridine etc., were used in solution polycondensation to remove $\mathrm{HCl}$, however additional processes were needed to separate triethylamine hydrochloride as a by-product. Moreover, the obtained PPDAs usually have a wide dispersity $(\nexists>4)$ by the reaction. ${ }^{32,33}$ In order to investigate the structure-property relationships, PPDAs with narrow molecular-weight distribution were desired. Thus, nitrogen atmosphere was utilized to remove the produced $\mathrm{HCl}$ instead of the acid-binding agent in the reaction. Gel permeation chromatography reveals the weight-average molecular weight $\left(M_{\mathrm{w}}\right)$ of POS, POM and POA of 6027,7611 and 8300, and the molecular weight dispersity $(\nexists)$ of 1.05, 1.17 and 1.12. The dispersity typically stems from side reactions, such as chlorination and alkylation of amino groups or reaction with residual water. ${ }^{22,42}$

The synthesized PPDAs were also characterized by ${ }^{1} \mathrm{H}$ NMR (Fig. 1) and ${ }^{31}$ P NMR (Fig. 2) spectroscopy. As for the PPDAs, the multiplied between 6.56 and $7.90 \mathrm{ppm}$ correspond to the protons of the benzene ring. The $\mathrm{CH}_{3}$ protons in POM appear at $3.63 \mathrm{ppm}$. In addition, a signal at $8.82 \mathrm{ppm}$ in POS representing the protons of the repeat imino group was observed. ${ }^{43}$ The ${ }^{31} \mathrm{P}$ NMR spectra of the PPDAs all show two signals; one around $8.46 \mathrm{ppm}$ corresponds to the phosphorus in the repeat unit and 


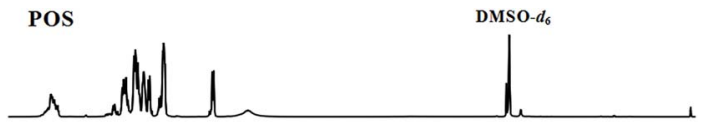

POM
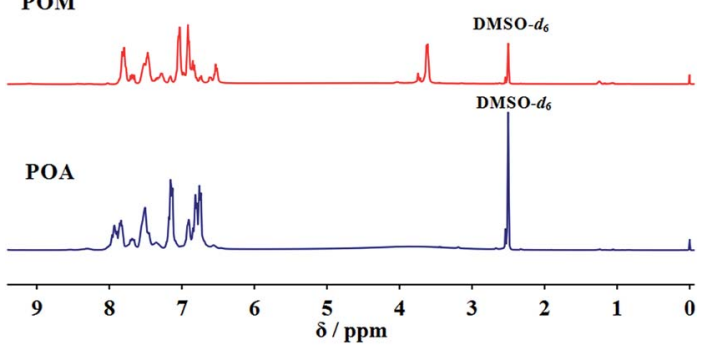

Fig. $1{ }^{1} \mathrm{H}$ NMR of the synthesized PPDAs.
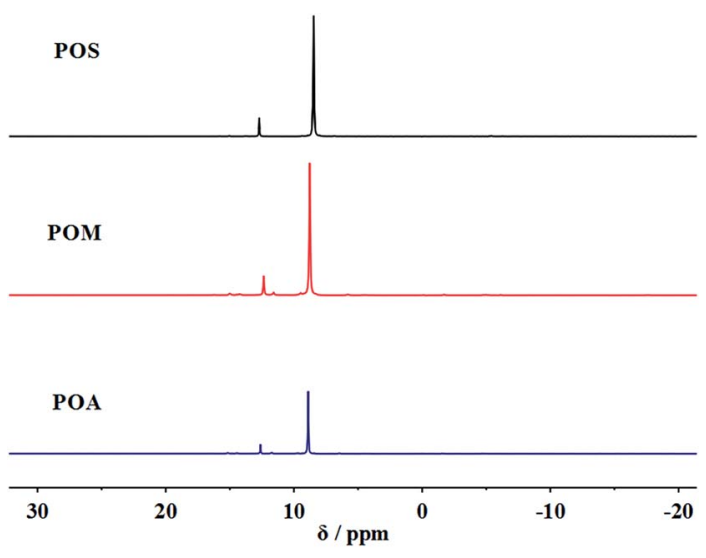

Fig. $2{ }^{31}$ P NMR of the synthesized PPDAs.

the other one around $12.61 \mathrm{ppm}$ to the phosphorus in the chain end. ${ }^{44}$ For example, the phosphorus in the repeat unit is attached to one phenyl group and two imino groups whereas that at the end is attached to one phenyl group, one imino group and one aryloxy group. ${ }^{44}$ All the spectroscopic data support the structure of PPDAs.
3.1.2 DSC of PPDAs. The influence of the incorporated backbone structure on the $T_{\mathrm{g}}$ values for the PPDAs was investigated by DSC. Fig. 3a shows the DSC curves of the PPDAs, and their $T_{\mathrm{g}}$ values are summarized in Table 1. It is evident that all the PPDAs have phase-transition behaviors with $T_{\mathrm{g}} \mathrm{s}$ ranging from 130 to $195{ }^{\circ} \mathrm{C}$, indicating that in the temperature range studied, no melting occurs and all the PPDAs display good thermal stability. As illustrated in Fig. 3a, POS achieves the highest glass transition temperature, equal to $191{ }^{\circ} \mathrm{C}$. This could be attributed to the incorporation of the sulfone group, which could increase the intermolecular forces. Generally, the $T_{\mathrm{g}}$ decreases with increasing flexibility of the molecules. The $T_{\mathrm{g}} \mathrm{S}$ of POM and POA are also in agreement with the principle. The trends of the polymers' $T_{\mathrm{g}} \mathrm{s}$ are also consistent with previous reports. $^{33}$

3.1.3 TGA of PPDAs. The TGA of the PPDAs under nitrogen atmosphere are shown in Fig. $3 \mathrm{~b}$. The temperatures corresponding to $5 \%$ weight loss $\left(T_{\text {onset }}\right)$ and to maximum decomposition rate $\left(T_{\max }\right)$ along with the char yield at $700^{\circ} \mathrm{C}$, are given in Table 1. As for the thermal degradation behavior of the PPDAs, POM and POA start to decompose at about $290{ }^{\circ} \mathrm{C}$ and undergo flat decomposition between 290 to $470{ }^{\circ} \mathrm{C}$, whereas POS decomposes in one sharp step with $T_{\max }=370{ }^{\circ} \mathrm{C}$. As for the char yield of the PPDAs at $700{ }^{\circ} \mathrm{C}$, POS achieves the highest char yield, as high as $48.8 \%$, which is more than the values exhibited by POM and POA, equal to $31.1 \%$ and $41.4 \%$, respectively. These results show that the incorporated sulfone groups could impact the thermal stability more than either the ether or methylene groups. The following order of thermal stability and char forming efficiencies of the incorporated groups were obtained: $-\mathrm{SO}_{2}->-\mathrm{O}->-\mathrm{CH}_{2}-$. Generally, it is

Table 1 Thermal properties of the PPDAs

\begin{tabular}{lllll}
\hline Sample & $T_{\mathrm{g}}\left({ }^{\circ} \mathrm{C}\right)$ & $T_{\text {onset }}\left({ }^{\circ} \mathrm{C}\right)$ & $T_{\max }\left({ }^{\circ} \mathrm{C}\right)$ & Char $\left(700{ }^{\circ} \mathrm{C}, \mathrm{wt} \%\right)$ \\
\hline POS & 191 & 341 & 370 & 48.8 \\
POM & 136 & 288 & 316,427 & 31.1 \\
POA & 133 & 293 & 315,437 & 41.4
\end{tabular}

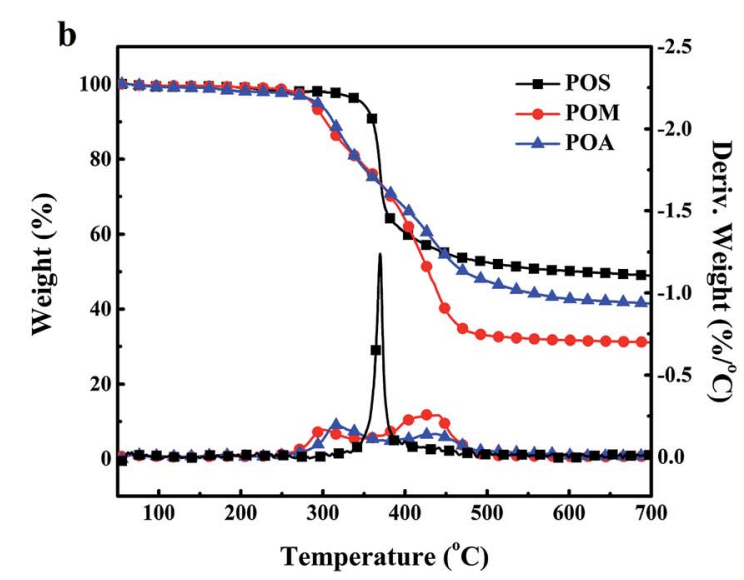

Fig. 3 DSC (a) and TGA (b) curves of the PPDAs.

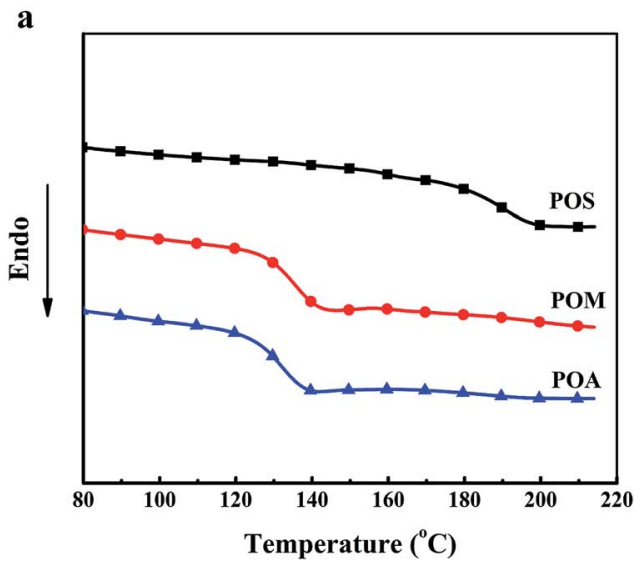

. 3 DSC (a) and TGA (b) curves of the PPDAs. 
considered that the thermal stability of the flame retardant is directly related to its flame retardancy. The high char yield suggests the PPDAs possess excellent thermal stability and are expected to achieve good flame retardancy. ${ }^{33}$

3.1.4 IR analysis of evolved gases from PPDAs. To better understand the thermal degradation behavior of the PPDAs, the pyrolysis gases formed during thermal degradation under nitrogen were studied by TGA-FTIR. The 3D curves of the TGAFTIR spectra for the PPDAs are shown in Fig. 4a, and the extracted FTIR curves of the PPDAs at the maximum decomposition temperature are displayed in Fig. $4 \mathrm{~b}$ and c. As can be observed from Fig. $4 \mathrm{~b}$, the main pyrolysis gases for the PPDAs could be identified as aromatic compounds $(3057,1610$ and $\left.1500 \mathrm{~cm}^{-1}\right)$ and $\mathrm{CO}_{2}\left(2355\right.$ and $\left.670 \mathrm{~cm}^{-1}\right) \cdot{ }^{26}$ Besides, the formation of benzene $\left(\mathrm{C}=\mathrm{C}\right.$ vibrations at 1946 and $\left.1801 \mathrm{~cm}^{-1}\right)$ as a result of the decomposition of aromatic compounds was confirmed. It is notable that the characteristic peak of PO compounds was observed at $1035 \mathrm{~cm}^{-1}$ as a weak signal, which may be due to the scission of the phosphonamide structure and play a role in the gaseous flame retardant effect. ${ }^{45}$ Furthermore, additional strong absorbances of sulfur-containing fragments for POS were found at 1375 and $1340 \mathrm{~cm}^{-1}$, which are attributed to the sulfone groups in the DDS structure. ${ }^{46}$ As for the second decomposition stage of POM and POA (Fig. 4c), the main pyrolysis gases were aromatic compounds (3055, 1616 and $\left.1508 \mathrm{~cm}^{-1}\right), \mathrm{CO}_{2}$ (2355 and $670 \mathrm{~cm}^{-1}$ ) and $\mathrm{PO}$ compound $\left(968 \mathrm{~cm}^{-1}\right)$, similar to those detected during the first decomposition stage. ${ }^{45}$ Besides, aromatic $\mathrm{C}-\mathrm{O}$ compounds $\left(1332 \mathrm{~cm}^{-1}\right)$ were identified as thermal decomposition products for POA.

To summarize, the decomposition of the PPDAs can be divided into two steps; the first step is scission of the pendant group and the main chain of the polymers, corresponding to a few of aromatic fragments, the other is pyrolysis and charring of the residue at higher temperature. This result was also agreed with the two decomposition steps observed in the TGA curves of POA and POM. As for the one-step decomposition of POS, this may be explained by the overlap of the two decomposition steps.

3.1.5 Flammability properties of PPDAs. The flammability of PPDAs was evaluated by PCFC, which could provide a novel and rapid method for measuring dynamic combustion parameters of milligram-sized samples. The heat release rate (HRR), heat release capacity (HRC) and total heat release (THR), which are calculated from the oxygen consumption history, are important parameters to determine the hazard potential of polymers in a fire scenario. The heat release curves of the PPDAs and detailed data are presented in Fig. 5 and Table 2, respectively.

As for POS, it exhibits a sharp heat release peak and gives the highest HRC value of $330 \mathrm{~J} \mathrm{~g}^{-1} \mathrm{~K}$. In the case of POM and POA, there are much broader heat release regions, between 250 to $600{ }^{\circ} \mathrm{C}$ and lower $\mathrm{HRC}$ values of 166 and $97 \mathrm{~J} \mathrm{~g}^{-1} \mathrm{~K}$, respectively,
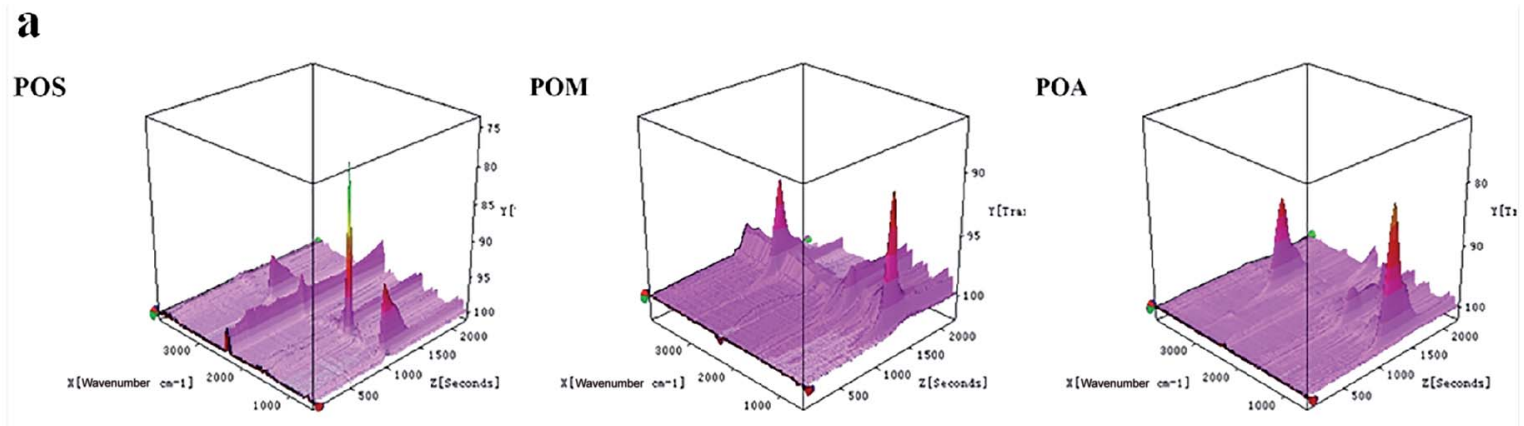

b

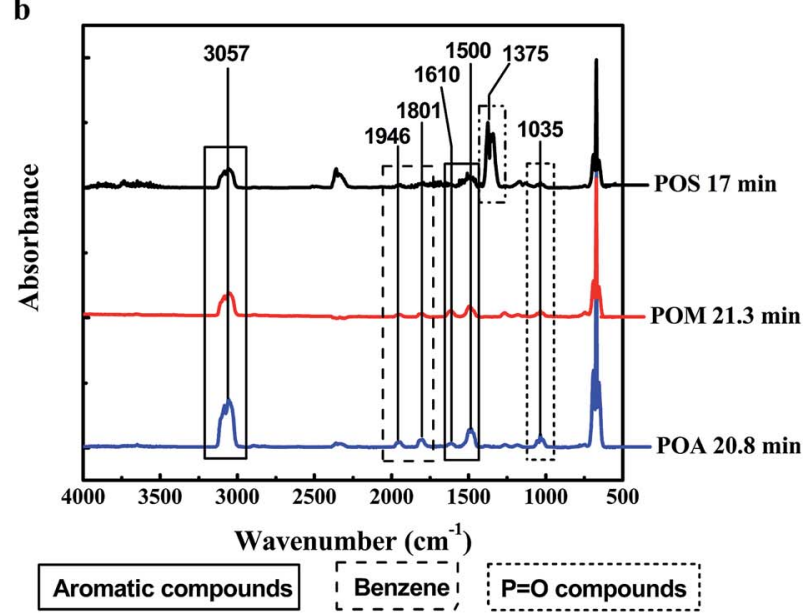

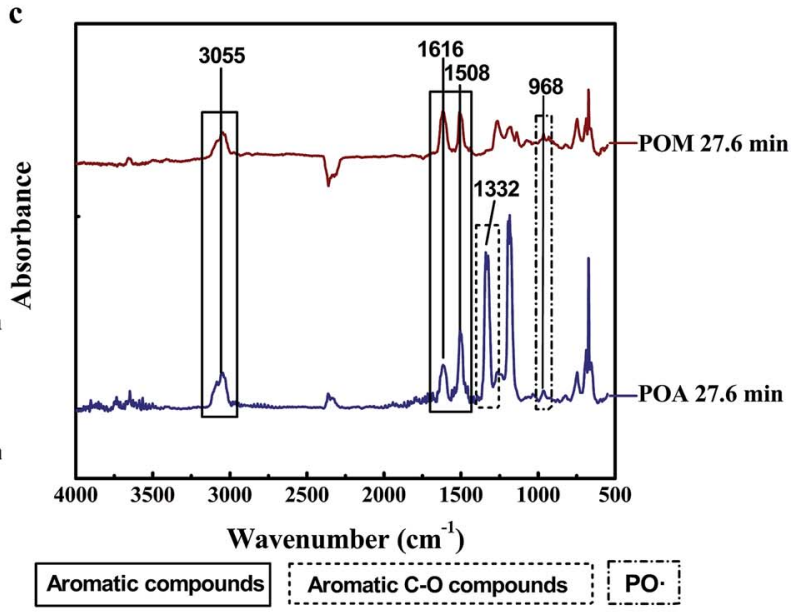

Fig. 4 3D curves of the TG-FTIR spectra for the PPDAs (a) and the extracted FTIR spectra of the evolved pyrolysis gas at $T_{\max 1}$ (b) and $T_{\max 2}$ (c). 


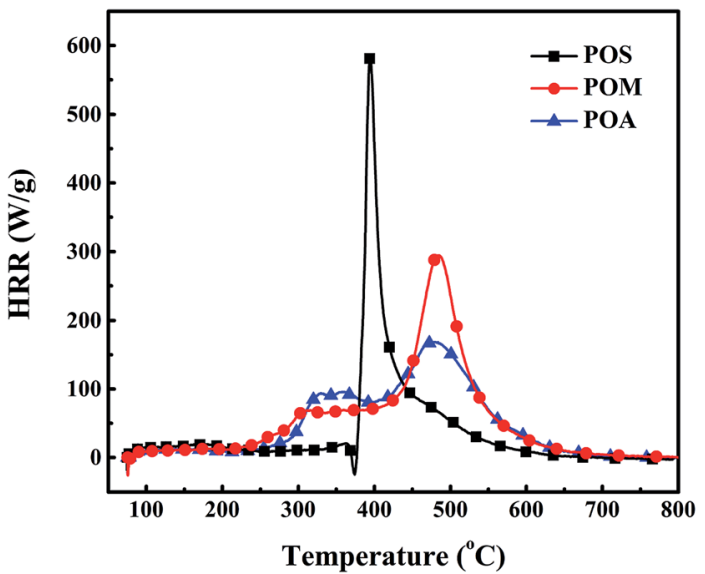

Fig. 5 The heat release rate curves of POS, POM and POA.

Table 2 PCFC data of POS, POM, POA and some other polymeric materials

\begin{tabular}{llll}
\hline Sample & HRC $\left(\mathrm{J} \mathrm{g}^{-1} \mathrm{~K}\right)$ & PHRR $\left(\mathrm{W} \mathrm{g}^{-1}\right)$ & THR $\left(\mathrm{kJ} \mathrm{g}^{-1}\right)$ \\
\hline POS & 330 & 582.6 & 15.4 \\
POM & 166 & 294.2 & 20.5 \\
POA & 97 & 165.2 & 18.5 \\
\hline
\end{tabular}

compared to POS, indicating lower flammability. Generally speaking, the HRC value is mainly decided by two factors: the maximum decomposition rate and the heat of combustion of the pyrolysis gases decomposed at the temperature. From Fig. 5, it can be observed that the sulfur-containing gases were released at the maximum decomposition rate, which leads to a higher HRC value. The PHRR values of the PPDAs are similar to their HRC values. It is worth noting that POS also exhibits the lowest THR value of $15.4 \mathrm{~kJ} \mathrm{~g}^{-1}$, while POM and POA give higher values of 20.5 and $18.5 \mathrm{~kJ} \mathrm{~g}^{-1}$, respectively. It directly demonstrates that the incorporation of $\mathrm{S}$ or $\mathrm{O}$ into the backbone of polymers could efficiently reduce its flammability. It seems that a polymer with more heteroatom as well as high char residue yields possesses lower flammability.

Above all, the synthesized PPDAs all possess high glass transition temperature, good thermal stability, high char yield and lower flammability, associated to their backbone structures. Aromatic compounds and phosphorus-containing pyrolysis gases were detected as products of the thermal decomposition of the PPDAs. An expected high efficiency in flame retardancy could be deduced by the good thermal properties, lower flammability and phosphorus-containing gases generated from the PPDAs.

\subsection{Thermal properties of EP and EP/PPDA composites}

3.2.1 DSC of EP and EP/PPDA composites. An important parameter for $\mathrm{EP}$ is the glass transition temperature, which has a strong influence on the material's potential applications; however, its value is often overlooked owing to the high loading and poor compatibility of flame retardants. The influence of the PPDAs on the $T_{\mathrm{g}}$ of the composites is displayed and summarized in Fig. 6 and Table 3. POS shows nearly negligible negative effects on the $T_{\mathrm{g}}$ of EP/POS. This may be explained by the good compatibility and possible cohesive forces (hydrogen bonds) between POS and EP molecular. On the other hand, it is found that $\mathrm{EP} / \mathrm{POM}$ and $\mathrm{EP} / \mathrm{POA}$ have lower $T_{\mathrm{g}} \mathrm{s}$ than $\mathrm{EP} / \mathrm{POS}$ and pure $\mathrm{EP}$, indicating a low plasticizer effect of these polymer, which is consistent with the results from previous work. ${ }^{11,29}$

3.2.2 TGA of EP and EP/PPDA composites. TGA and DTG curves of EP and EP/PPDA composites provided information about their thermal stabilities and thermal degradation behaviors. Fig. 7 shows the TGA and DTG curves of samples under nitrogen, and detailed data were collected in Table 3. Pure EP started to decompose at $T_{\text {onset }}=367^{\circ} \mathrm{C}$. As for EP/PPDA composites, $T_{\text {onset }}$ and $T_{\max }$ are all shifted to lower temperatures compared to $\mathrm{EP}$, due to the earlier decomposition and catalysis effect of PPDAs on EP. With the addition of PPDAs, more char residue was formed at the end of decomposition step. The increase in char residue was greater than the calculated value based on the superposition of the char yields of single components. A reaction between PPDAs and EP is concluded, enhancing the char amount. More importantly, it is noted that the TGA and DTG curves of EP/PPDA composites were similar to each other, suggesting a strong relationship between the chemical environment of the phosphorus in PPDAs and thermal stability of EP substrate.

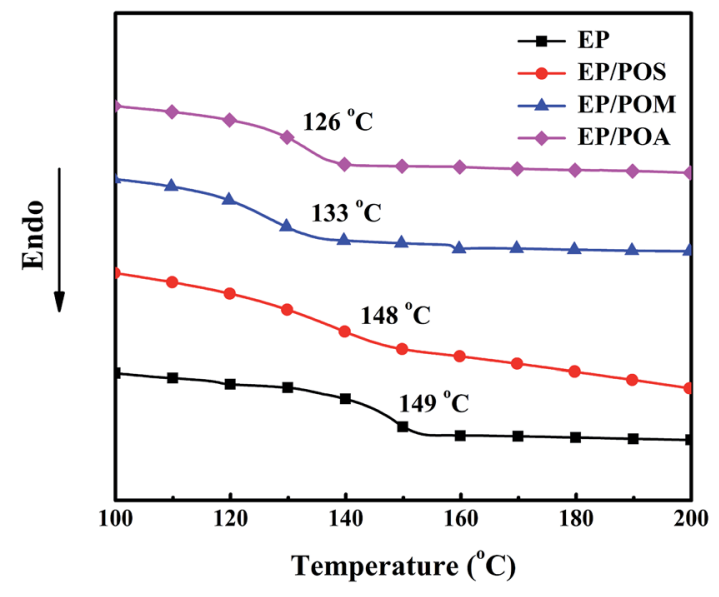

Fig. 6 DSC curves of EP and EP/PPDA composites.

Table 3 Thermal properties of EP and EP/PPDA composites

\begin{tabular}{lllll}
\hline Sample & $T_{\mathrm{g}}\left({ }^{\circ} \mathrm{C}\right)$ & $T_{\text {onset }}\left({ }^{\circ} \mathrm{C}\right)$ & $T_{\max }\left({ }^{\circ} \mathrm{C}\right)$ & Char $^{a}$ Exp./Calcd. \\
\hline EP & 149 & 367 & 379 & $16.5 /-$ \\
EP/POS & 148 & 318 & 339 & $21.7 / 21.3$ \\
EP/POM & 133 & 301 & 326 & $20.1 / 18.7$ \\
EP/POA & 126 & 296 & 327 & $21.5 / 20.2$
\end{tabular}

${ }^{a}$ Char yield at $700{ }^{\circ} \mathrm{C}$. 

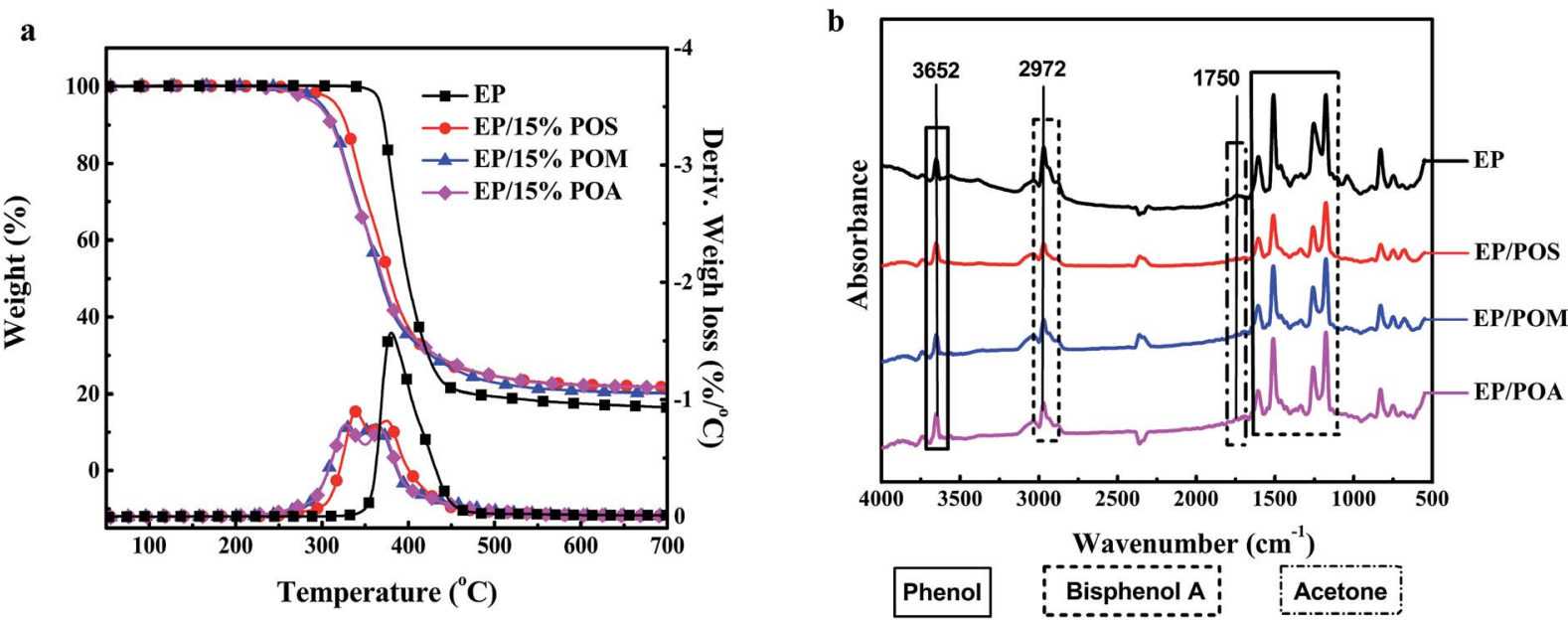

Fig. 7 TGA and DTG curves of EP and EP/PPDA composites under nitrogen (a), FTIR spectra of the evolved pyrolysis gas at $T_{\text {max }}$ (b).

3.2.3 Thermal decomposition behaviors of EP and EP/ PPDA composites. To better investigate the thermal degradation behavior of EP and EP/PPDA composites, the pyrolysis gases during thermal decomposition were analyzed by TGAFTIR (Fig. 7b). During the main decomposition step, phenolic derivatives and bisphenol A were monitored with the characteristic peaks at $3652 \mathrm{~cm}^{-1}$ assigned to the aromatic $\mathrm{OH}$ stretch, and the aromatic $\mathrm{C}=\mathrm{C}$ vibrations at $1609 \mathrm{~cm}^{-1}$. The peak at $1338 \mathrm{~cm}^{-1}$ is the typical absorption bands of aromatic C-O stretch of phenol and bisphenol A. The peak at $2972 \mathrm{~cm}^{-1}$ attribute the $\mathrm{CH}_{3}$ stretch of bisphenol A. Further, the broad peak at $1750 \mathrm{~cm}^{-1}$ and the strong peak at $1250 \mathrm{~cm}^{-1}$ confirm the presence of acetone as thermal decomposition products of EP and EP/PPDA composites. ${ }^{25}$ Furthermore, the weak peak at $3386 \mathrm{~cm}^{-1}$ is proposed as decomposition products of the curing agent $m$-PDA and PPDAs.

As for the EP/PPDA composites, the characteristic peaks of PPDAs such as $\mathrm{P}=\mathrm{O}$ stretch at $1045 \mathrm{~cm}^{-1}$ and $\mathrm{P}-\mathrm{O}$ stretch at $887 \mathrm{~cm}^{-1}$ of phosphates, phosphites, and phosphoric acid derivatives were detected as a weak signal and shifted in comparison with PPDAs, indicating a change in the chemical environment. The absence of phenol, bisphenol $\left(3652 \mathrm{~cm}^{-1}\right.$, $\left.1609 \mathrm{~cm}^{-1}\right)$, and acetone $\left(1750 \mathrm{~cm}^{-1}, 1250 \mathrm{~cm}^{-1}\right)$ remained in comparison to EP. ${ }^{25}$ Besides, the reduced absorbance of flammable gases (acetone and bisphenol A) (Fig. 7b) and increased char residue at $700{ }^{\circ} \mathrm{C}$ demonstrate the reactions between PPDAs and EP, indicating the probable gas-phase activity of the PPDAs in flame retardancy.

\subsection{Flame retardant properties of EP and EP/PPDA composites}

3.3.1 LOI and UL-94 tests. The flame retardancy of EP/ PPDA composites was evaluated using LOI and UL-94 vertical burning tests. Fig. 8 gives photographs of the char residue of EP/ PPDA composites at the end of LOI tests. Pure EP only achieve a poor char residue. With the addition of PPDAs, the char residue from EP/PPDA composites increased remarkably. The

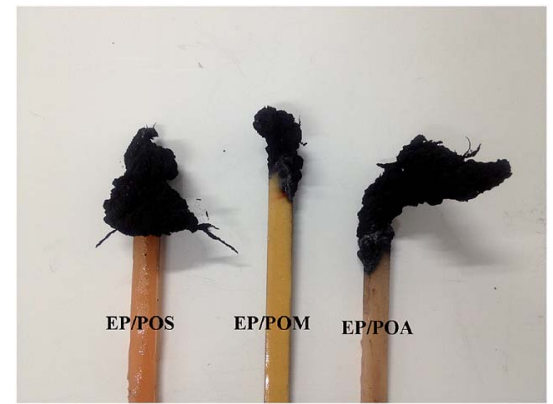

Fig. 8 Digital photos of char residue of EP/PPDA composites after LOI tests.

char residue can protect the matrix and reduce the heat transfer to pyrolysis zone and flammable gases transfer to the flame zone.

As shown in Table 4, pure EP had no rating in UL-94 vertical tests, and a LOI value is $24.7 \%$. For EP/PPDA composites, the LOI value increased with the incorporation of the PPDAs. While the LOI value of EP/POM increased to $28.9 \%$, the UL-94 rating achieved a V-1 rating. When $15 \mathrm{wt} \%$ POS or POA was incorporated into $\mathrm{EP}$, the UL-94 test achieved a V-0 rating, indicating a significant contribution of the heteroatom towards obtaining a reduced flammability. Obviously, the PPDAs are a series of efficient mono-component flame retardant for EP.

Table $4 \mathrm{LOI}$ and UL-94 testing results of EP and EP/PPDA composites

\begin{tabular}{llllll}
\hline & & \multicolumn{2}{l}{ UL-94 } & & \\
\cline { 3 - 6 } Sample & LOI $(\%)$ & $t_{1}(\mathrm{~s})$ & $t_{2}(\mathrm{~s})$ & Dripping & Rating \\
\hline EP & 24.7 & Infinite & - & No & No rating \\
EP/POS & 29.5 & 6 & 3 & No & V-0 \\
EP/POM & 28.9 & 19 & 36 & No & V-1 \\
EP/POA & 29.6 & 9 & 1 & No & V-0
\end{tabular}


3.3.2 Cone calorimeter test. Cone calorimeter test was performed to investigate the flame retardant properties of PPDAs in EP. The concerned combustion parameters such as time to ignition $\left(t_{\text {ign }}\right)$, total heat release (THR), peak of heat release rate (PHRR), peak of total mass loss (TML), average of HRR (av-HRR), average of effective heat of combustion (av-EHC) and total heat release per total mass loss (THR/TML) are listed in Table 5. Once ignition, pure EP burned rapidly and exhibited a poor and limited char residue. With the addition of PPDAs, the samples expanded faster and more strongly, yielded about $21 \mathrm{wt} \%$ of char residue. The shapes of the heat release rate (HRR) curves are similar for EP and EP/PPDA composites (Fig. 9a). Shortly after ignition, the HRR reached maximum peak, since a protection layer was formed decreasing the HRR. Meanwhile, the time to ignition $\left(t_{\text {ign }}\right)$ of EP/PPDA composites was earlier than pure EP, which is corresponded to the catalysis effect of PPDA on the char-forming process for the composites. As shown in Table 5, the PHRR and THR value of EP/PPDA composites, were reduced by approximately $47 \%$ and $30 \%$, respectively. The av-HRR for all investigated materials are ordered by reducing the fire risk: EP/POS $(34 \%)<\mathrm{EP} / \mathrm{POA}(30 \%)$ $<$ EP/POM $(24 \%)<$ EP. The tendency in HRR curves for EP and EP/PPDA composites was similar to the TGA curves, indicating the flame retardancy of flame retarded EP may associate with the chemical environment of the phosphorus in the flame retardant to some extent. Moreover, after ignition, the THR curve for EP/POS was much lower than those for EP/POM and
EP/POA for all times ranging between 75 and $200 \mathrm{~s}$ (Fig. 9b). At the fire growth period of real fire conditions, lower heat release means a lower rate of flame spreading and a greater time available for response.

The THR divided by the total mass loss (THR/TML) equals the product between effective heat of combustion of the volatiles and the combustion efficiency. ${ }^{25}$ The THR/TML ratio for EP/PPDA composites was reduced by $16 \%$ due to flame inhibition. Moreover, monitoring the $\mathrm{CO} / \mathrm{CO}_{2}$ ratio (Fig. 10) during cone calorimeter tests shows the peak $\mathrm{CO} / \mathrm{CO}_{2}$ ratios of $\mathrm{EP} / \mathrm{POS}$, $\mathrm{EP} / \mathrm{POM}$, and EP/POA increased by $212 \%, 174 \%$ and $211 \%$ over EP, respectively. Thus, PPDAs in EP worked in the gas phase as well as in the condensed phase.

In order to evaluate the flame retardant effect quantitatively, the main flame retardant modes of action were calculated according to eqn (1)-(3). ${ }^{47,48}$ The results are listed in Table 6 . With the addition of PPDAs, the flame inhibition effect, charring effect, and barrier and protective effect of EP/PPDA composites all sharply increased compared with EP. It can be concluded that the flame inhibition effect of PPDAs is composed of the radical quenching effect and the dilution effect of volatile phosphorus-based pyrolysis gases. The best results in terms of increasing charring effect and barrier and protective effect were achieved by EP/POS compared to EP/POM and EP/ POA, indicating that sulfone group in PPDA were more efficient in condensed phase action than methyl group and ether group. It is also noted that EP/POA showed a better flame

Table 5 Cone calorimeter results for EP and EP/PPDA composites

\begin{tabular}{|c|c|c|c|c|c|c|}
\hline Sample & $\mathrm{THR}\left(\mathrm{MJ} \mathrm{m} \mathrm{m}^{-2}\right)$ & PHRR $\left(\mathrm{kW} \mathrm{m}^{-2}\right)$ & TML (wt\%) & av-EHC (MJ kg $\left.{ }^{-1}\right)$ & $\begin{array}{l}\text { THR/TML } \\
\left(\mathrm{MJ} \mathrm{m} \mathrm{m}^{-2} \mathrm{~g}^{-1}\right)\end{array}$ & $\begin{array}{l}\text { av-HRR } \\
\left(\mathrm{kW} \mathrm{s} \mathrm{s}^{-1} \mathrm{~m}^{-2}\right)\end{array}$ \\
\hline EP & $77 \pm 10$ & $944 \pm 88$ & $95.2 \pm 0.7$ & $16.1 \pm 0.8$ & $3.7 \pm 0.1$ & $233 \pm 25$ \\
\hline EP/15\% POS & $54 \pm 3$ & $462 \pm 25$ & $78.8 \pm 0.7$ & $13.5 \pm 0.4$ & $3.1 \pm 0.1$ & $154 \pm 20$ \\
\hline $\mathrm{EP} / 15 \% \mathrm{POM}$ & $54 \pm 2$ & $508 \pm 10$ & $79.4 \pm 0.1$ & $13.6 \pm 0.5$ & $3.1 \pm 0.1$ & $176 \pm 20$ \\
\hline $\mathrm{EP} / 15 \% \mathrm{POA}$ & $52 \pm 2$ & $492 \pm 20$ & $79.5 \pm 0.2$ & $12.7 \pm 0.5$ & $3.0 \pm 0.1$ & $163 \pm 11$ \\
\hline
\end{tabular}
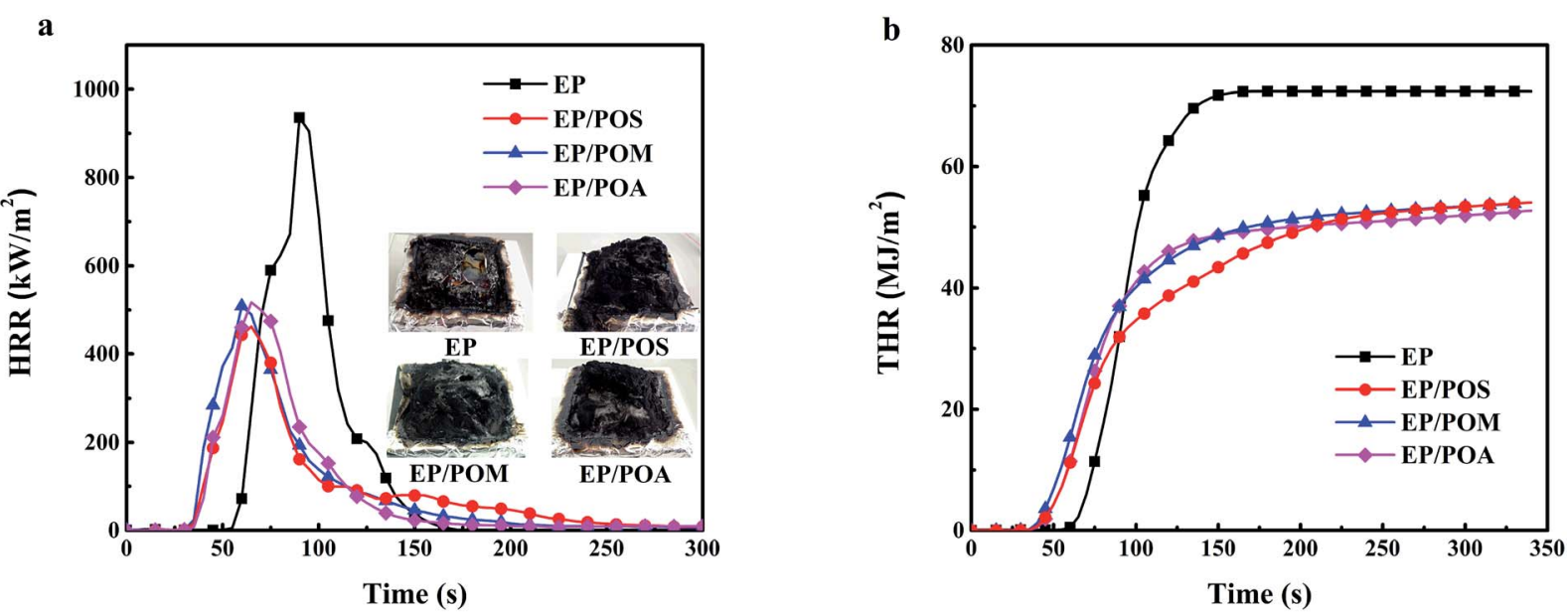

Fig. 9 Cone calorimeter results: (a) heat release rate (HRR) of EP and EP/PPDA composites versus time, (b) total heat release (THR) of EP and EP/ PDPA composites versus time. 


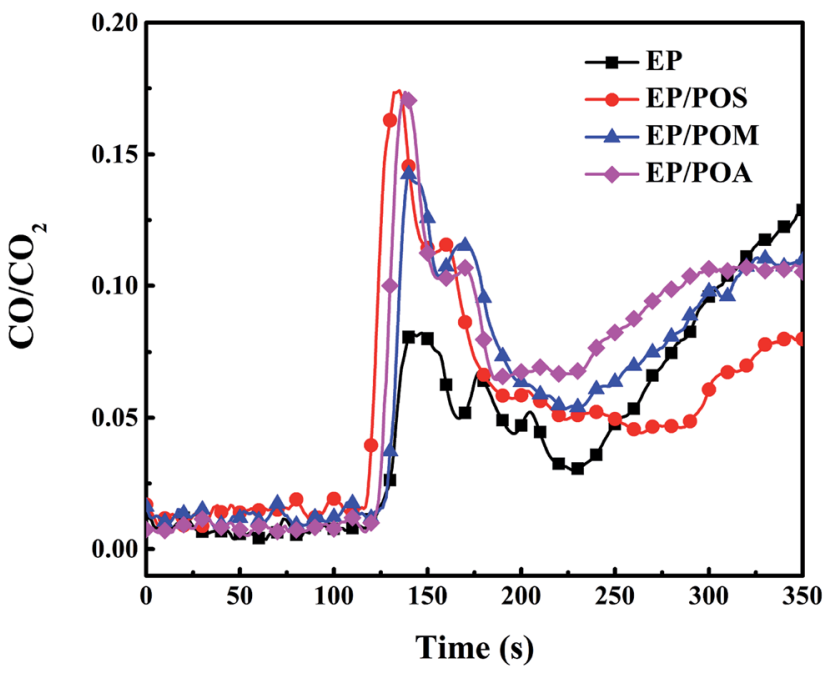

Fig. $10 \mathrm{CO} / \mathrm{CO}_{2}$ weight ratio as a function of time during cone calorimeter tests for EP and EP/PPDA composites.

Table 6 Quantitative assessment of the main flame retardant modes of action

\begin{tabular}{llll}
\hline Samples & $\begin{array}{l}\text { Flame inhibition } \\
\text { effect }\end{array}$ & $\begin{array}{l}\text { Charring } \\
\text { effect }\end{array}$ & $\begin{array}{l}\text { Barrier and } \\
\text { protective effect }\end{array}$ \\
\hline EP/POS & $16 \%$ & $17.2 \%$ & $30 \%$ \\
EP/POM & $15 \%$ & $16.6 \%$ & $23 \%$ \\
EP/POA & $21 \%$ & $16.5 \%$ & $23 \%$
\end{tabular}

inhibition effect than EP/POS and EP/POM, which may correspond to more phosphorus contents were released into gas phase. The protective barrier effect combined with flame inhibition effect plays a dominant role in the flame retardant performance of PPDAs in EP.

$$
\begin{gathered}
\text { Flame inhibition effect }=1-\mathrm{EHC}_{\mathrm{EP} / \mathrm{PPDA}} / \mathrm{EHC}_{\mathrm{EP}} \\
\text { Charring effect }=1-\mathrm{TML}_{\mathrm{EP} / \mathrm{PPDA}} / \mathrm{TML} \mathrm{EP}_{\mathrm{EP}}
\end{gathered}
$$

Barrier and protective effect $=1-\left(\mathrm{PHRR}_{\mathrm{EP} / \mathrm{PPDA}} / \mathrm{PHRR}_{\mathrm{EP}}\right) /$

$$
\left(\mathrm{THR}_{\mathrm{EP} / \mathrm{PPDA}} / \mathrm{THR}_{\mathrm{EP}}\right)
$$

3.3.3 Element analysis of char residue after cone tests. The element contents on the external and internal char layer from EP and EP/PPDA composites after the cone tests was investigated by XPS. The results are listed in Table 7. In both external and internal char layer obtained from cone tests, C, N, O and P elements were detected. The phosphorus and oxygen contents in the external char layer are higher than that from samples of internal char layer, indicating a better oxidation resistance of the protective char layer. More importantly, the extremely low phosphorus content in the internal char layer prove the release of majority of the phosphorus into the flame zone, forming phosphorus-based free radicals to perform the flame inhibition effect in the gas phase..$^{22}$ As for EP/POS composites, the higher
Table 7 XPS data of the char residue

\begin{tabular}{llllll}
\hline Samples & $\mathrm{C}$ & $\mathrm{N}$ & $\mathrm{O}$ & $\mathrm{P}$ & $\mathrm{S}$ \\
\hline EP/POS external & 75.98 & 4.33 & 17.68 & 1.33 & 0.69 \\
EP/POS internal & 80.34 & 3.67 & 14.04 & 0.56 & 1.38 \\
EP/POM external & 76.55 & 5.99 & 16.16 & 1.30 & - \\
EP/POM internal & 82.01 & 4.35 & 12.93 & 0.70 & - \\
EP/POA external & 76.03 & 5.45 & 16.92 & 1.61 & - \\
EP/POA internal & 82.00 & 3.86 & 13.91 & 0.23 & - \\
\hline
\end{tabular}

sulfur content in the internal surface of char residue can be explained by the low-volatile sulfur-based decomposition product of POS in the condensed phase. It is reported that the sulfur-based products could accelerate the oxidation reaction an formation of char precursors. ${ }^{49}$ Besides, the results also show that there are a synergistic effect between the heteroatom and the phosphonamide structure.

\subsection{Pyrolysis behaviors of POS}

The pyrolysis behavior of POS was investigated by pyrolysis-GC/ MS. In this part of the study, POS was selected to be representative sample of PPDAs. The recorded total ion chromatogram and the speculative decomposition route is presented in Fig. 11. The ion fragments, such as $66,78 \mathrm{~m} / \mathrm{z}$ for benzene and $94 \mathrm{~m} / \mathrm{z}$ for aminobenzene, is mainly attributed to the decomposition products of phosphonamide and diphenyl sulfone structure. ${ }^{41}$ The peaks at 47,63 , and $94 \mathrm{~m} / z$ were assigned to $\mathrm{PO}, \mathrm{PO}_{2}$ and $\mathrm{P}_{2} \mathrm{O}_{2}$ compound, which could act radical-scavenging mechanism during combustion. ${ }^{41,50}$ Speculative decomposition route of POS is shown in Fig. 11b. Phosphorous acid $\left(\mathrm{H}_{3} \mathrm{PO}_{3}\right)$ was supposed to promote the char forming process in the condensed phase.

\subsection{Flame retardant mechanism}

Combining the analysis in Section 3.2.3 and 3.3 about thermal decomposition behaviors and flame retardant properties of EP and EP/PPDA composites with 3.4 pyrolysis behaviors of POS, flame retardant mechanism of PPDAs in EP are proposed as below.

In the condensed phase, PPDAs promote the decomposition and char-forming processes of EP at a low temperature. The acid compounds $\left(\mathrm{H}_{3} \mathrm{PO}_{3}\right)$ from the thermal decomposition of PPDAs could also promote the char-forming process of EP matrix and the formation of protective char layers. The char yield observed in the cone test was increased from $4.8 \mathrm{wt} \%$ for EP up to about $21 \mathrm{wt} \%$ for EP/PPDA composites as evidence. The phosphorus and oxygen contents in the external char layer are higher than that from samples of internal char layer, indicating a better oxidation resistance of the protective char layer. The phosphorus-containing char layer could act as barrier to hinder the exchange of fuel, oxygen and heat between EP matrix and fire zone.

In the gas phase, PPDAs in EP showed obvious flame inhibition effect during combustion. As seen from Fig. 11, the pyrolysis behaviors of POS confirmed the presence of 


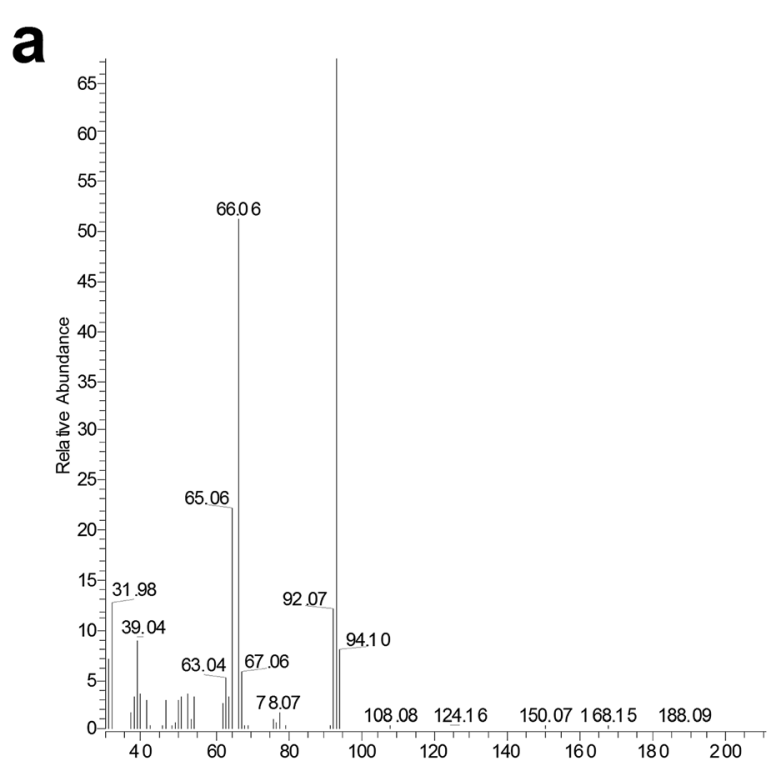

b

.

Fig. 11 Total ion chromatogram of POS (a) and speculative decomposition route of POS (b).

phosphorus-containing radicals in the decomposition route. As shown in Table 5 , the reduced combustion efficiency also demonstrate the flame inhibition effect of PPDAs in the gas phase. The phosphorus-containing radicals could interrupt the chain reaction during combustion of EP.

Above all, the flame retardant mechanism of PPDAs in EP could be summarized as follows: char forming effect and barrier and protective effect in the condensed phase, flame inhibition effect in the gas phase. Moreover, as shown in Table 6, the heteroatom in PPDAs have slightly influence on the flame retardant mechanism of EP/PPDA composites, such as barrier and protective effect and flame inhibition effect.

\subsection{Fracture toughness of EP and EP/PPDA composites}

The quasi-static fracture toughness values, $K_{\mathrm{IC}}$, of EP and EP/ PPDA composites are present in Table 8. As expected, the fracture toughness of EP/POS resulted in a moderate increase in $K_{\mathrm{IC}}$. In previous research, it is reported that phosphorus-containing polysulfone could act as toughness modifier for EP. ${ }^{51}$ The sulfone group in POS could endow EP with high fracture toughness. For EP/POM and EP/POA, the incorporation of POM and POA did not showed a serious deterioration on fracture toughness of EP, which may be explained by the decrease of crosslinking density of EP. Thus, the incorporation of PPDAs

Table 8 Fracture toughness data of EP and EP/PPDA composites

\begin{tabular}{ll}
\hline Samples & $K_{\mathrm{IC}}\left(\mathrm{MPa} \mathrm{m}^{1 / 2}\right)$ \\
\hline EP & $0.89 \pm 0.05$ \\
EP/POS & $0.93 \pm 0.06$ \\
EP/POM & $0.79 \pm 0.05$ \\
EP/POA & $0.83 \pm 0.08$
\end{tabular}

into EP showed some promising improvements in flame retardancy as well as slightly influence on fracture toughness.

\section{Conclusions}

A series of PPDAs with different backbone structure were successfully synthesized and well characterized. The thermal properties, thermal degradation behaviors and flammability were investigated. The results showed that the PPDAs exhibit high thermal stability, high glass transition temperature and low flammability, associated to their backbone structures. The following order of thermal stability and char forming efficiencies of the incorporated groups were obtained: $-\mathrm{SO}_{2}->-\mathrm{O}->$ $-\mathrm{CH}_{2}-$. From TGA-FTIR results, the pyrolysis gases of the PPDAs have been found to be mainly aromatic compounds and phosphorus-containing gases. The high char yield, low flammability, generated phosphorus-containing gases indicated the potential high flame retardancy of the PPDAs.

Cone calorimeter, LOI and UL-94 vertical burning tests all demonstrated the addition of PPDAs into EP have greatly enhanced flame retardancy of the composites. Due to the incorporation of heteroatom, POS and POA show better flame retardant performances in LOI and UL-94 tests than POM. Meanwhile, EP/POS shows a similar $T_{\mathrm{g}}$ with pure EP, which is attributed to the hydrogen-bond interaction. The heat release rate and thermal properties of flame retardant EP were highly depend on the chemical surrounding of phosphorus atom in PPDAs. The flame retardant mechanism of PPDAs in EP can be devices by the charring effect, barrier and protective effect in the condensed phase, and the flame inhibition effect in the gas phase. In addition, the incorporation of heteroatom into PPDAs could enhance the flame retardancy of EP composites, such as the sulfone group in promoting char forming process and the ether group in flame inhibition effect in gas phase. This paper 
might promote a directed design of new composites with high flame retardancy, glass transition temperature and fracture toughness for future applications, and provide a rapid approach to determine the flame retardancy of novel flame retardant.

\section{Conflicts of interest}

There are no conflicts to declare.

\section{Acknowledgements}

Financial supported by the Prospective Project of Technology and Engineering Center for Space Utilization, CAS, Grant No. CSU-QZKT-201708, and the joint fund of Key Research Program of Frontier Sciences, CAS, Grant No. QYZDB-SSW-JSC050.

\section{References}

1 B. Schartel, B. Perret, B. Dittrich, M. Ciesielski, J. Krämer, P. Müller, V. Altstädt, L. Zang and M. Döring, Macromol. Mater. Eng., 2016, 301, 9-35.

2 S. L. Qiu, X. Wang, B. Yu, X. M. Feng, X. W. Mu, R. K. K. Yuen and Y. Hu, J. Hazard. Mater., 2017, 325, 327-339.

3 E. N. Kalali, X. Wang and D. Y. Wang, Ind. Eng. Chem. Res., 2016, 55, 6634-6642.

4 Y. Qiu, L. J. Qian and W. Xi, RSC Adv., 2016, 6, 56018-56027. 5 P. Müller, M. Morys, A. Sut, C. Jäger, B. Illerhaus and B. Schartel, Polym. Degrad. Stab., 2016, 130, 307-319.

6 W. J. Liang, B. Zhao, P. H. Zhao, C. Y. Zhang and Y. Q. Liu, Polym. Degrad. Stab., 2017, 135, 140-151.

7 C. Ma, B. Yu, N. N. Hong, Y. Pan, W. Z. Hu and Y. Hu, Ind. Eng. Chem. Res., 2016, 55, 10868-10879.

8 G. M. Wu, B. Schartel, H. Bahr, M. Kleemeier, D. Yu and A. Hartwig, Combust. Flame, 2012, 159, 3616-3623.

9 B. Perret, B. Schartel, K. Stöß, M. Ciesielski, J. Diederichs, M. Döring, J. Krämer and V. Altstädt, Macromol. Mater. Eng., 2011, 296, 14-30.

10 M. Rakotomalala, S. Wagner and M. Döring, Materials, 2010, 3, 4300-4327.

11 C. Klinkowski, S. Wagner, M. Ciesielski and M. Döring, Polym. Degrad. Stab., 2014, 106, 122-128.

12 N. N. Tian, J. Gong, X. Wen, K. Yao and T. Tang, RSC Adv., 2014, 4, 17607-17614.

13 S. M. Unlu, S. D. Dogan and M. Dogan, Polym. Adv. Technol., 2014, 25, 769-776.

14 C. Gérard, G. Fontaine and S. Bourbigot, Materials, 2010, 3, 4476-4499.

15 E. N. Kalali, X. Wang and D. Y. Wang, J. Mater. Chem. A, 2015, 3, 6819-6826.

16 X. Wang, E. N. Kalali and D. Y. Wang, ACS Sustainable Chem. Eng., 2015, 3, 3281-3290.

17 E. N. Kalali, X. Wang and D. Y. Wang, Ind. Eng. Chem. Res., 2016, 55, 6634-6642.

18 Y. Liu, H. V. Babu, J. Q. Zhao, A. Goñi-Urtiaga, R. Sainz, R. Ferritto, M. Pita and D. Y. Wang, Composites, Part B, 2016, 89, 108-116.
19 J. T. Luo, S. R. Yang, L. Q. Lei, J. Q. Zhao and Z. Tong, Composites, Part A, 2017, 100, 275-284.

20 X. M. Zhao, D. Xiao, J. P. Alonso and D. Y. Wang, Mater. Des., 2017, 114, 623-632.

21 C. Ma, B. Yu, N. N. Hong, Y. Pan, W. Z. Hu and Y. Hu, Ind. Eng. Chem. Res., 2016, 55, 10868-10879.

22 T. C. Mauldin, M. Zammarano, J. W. Gilman, J. R. Shields and D. J. Boday, Polym. Chem., 2014, 5, 5139-5146.

23 H. M. Stapleton, S. Klosterhaus, A. Keller, P. L. Ferguson, S. Van Bergen, E. Cooper, T. F. Webster and A. Blum, Environ. Sci. Technol., 2011, 45, 5323-5331.

24 R. M. Perez, J. K. W. Sandler, V. Altstädt, T. Hoffmann, D. Pospiech, M. Ciesielski, M. Döring, U. Braun, A. I. Balabanovich and B. Schartel, Polymer, 2007, 48, 778-790.

25 K. Täuber, F. Marsico, F. R. Wurm and B. Schartel, Polym. Chem., 2014, 5, 7042-7053.

26 X. Wang, Y. Hu, L. Song, W. Y. Xing, H. D. Lu, P. Lv and G. X. Jie, Polymer, 2010, 51, 2435-2445.

27 X. Wang, L. Song, W. Y. Xing, H. D. Lu and Y. Hu, Mater. Chem. Phys., 2011, 125, 536-541.

28 L. Chen and Y. Z. Wang, Materials, 2010, 3, 4746-4760.

29 W. Zhao, J. P. Liu, Y. Zhang and D. M. Ban, RSC Adv., 2015, 5, 80415-80423.

30 H. Y. Ma and Z. P. Fang, Thermochim. Acta, 2012, 543, 130-136. 31 P. A. Song, H. Liu, Y. Shen, B. X. Du, Z. P. Fang and Y. Wu, J. Mater. Chem., 2009, 19, 1305-1313.

32 W. Zhao, J. P. Liu, H. Peng, J. Y. Liao and X. J. Wang, Polym. Degrad. Stab., 2015, 118, 120-129.

33 Q. L. Tai, Y. Hu, R. K. K. Yuen, L. Song and H. D. Lu, J. Mater. Chem., 2011, 21, 6621-6627.

34 M. Steinmann, M. Wagner and F. R. Wurm, Chemistry, 2016, 22, 17329-17338.

35 Z. Li, P. Wei, Y. Yang, Y. G. Yan and D. Shi, Polym. Degrad. Stab., 2014, 110, 104-112.

36 J. M. Park, J. Y. Lee and Y. H. Park, Macromol. Res., 2010, 18, 539-544.

37 M. Papanastasiou, A. W. McMahon, N. S. Allen, A. M. Doyle, B. J. Johnson and K. Keck-Antoine, Polym. Degrad. Stab., 2006, 91, 2675-2682.

38 U. Braun, A. I. Balabanovich, B. Schartel, U. Knoll, J. Artner, M. Ciesielski, M. Döring, R. Perez, J. K. W. Sandler and V. Altstädt, Polymer, 2006, 47, 8495-8508.

39 R. M. Perez, J. K. W. Sandler, V. Altstädt, T. Hoffmann, D. Pospiech, J. Artner, M. Ciesielski, M. Döring, A. I. Balabanovich, U. Knoll, U. Braun and B. Schartel, J. Appl. Polym. Sci., 2007, 105, 2744-2759.

40 J. Jing, Y. Zhang, X. L. Tang and Z. P. Fang, RSC Adv., 2016, 6, 49019-49027.

41 X. M. Zhao, H. V. Babu, J. Llorca and D. Y. Wang, RSC Adv., 2016, 6, 59226-59236.

42 W. Vogt and S. Balasubramanian, Macromol. Chem., 1973, 163, 111-134.

43 X. Wang, W. Y. Xing, X. M. Feng, B. Yu, L. Song and Y. Hu, Polym. Chem., 2014, 5, 1145-1154.

44 K. S. Annakutty and K. Kishore, Polymer, 1988, 29, 756-761. 45 L. J. Qian, L. J. Ye, Y. Qiu and S. R. Qu, Polymer, 2011, 52, 5486-5493. 
46 F. Samperi, C. Puglisi, T. Ferreri, R. Messina, G. Cicala, A. Recca, C. L. Restuccia and A. Scamporrino, Polym. Degrad. Stab., 2006, 92, 1304-1315.

47 S. Brehme, T. Köppl, B. Schartel and V. Altstädt, e-Polym., 2014, 14, 193-208.

48 T. Shuo, V. Wachtendorf, P. Klack, L. J. Qian, Y. P. Dong and B. Schartel, RSC Adv., 2017, 7, 720-728.
49 M. Lewin, J. Brozek and M. M. Martens, Polym. Adv. Technol., 2002, 13, 1091-1102.

50 B. Schartel, Materials, 2010, 3, 4710-4745.

51 R. M. Perez, J. K. W. Sandler, V. Altstädt, T. Hoffmann, D. Pospiech, M. Ciesielski, M. Döring, U. Braun, A. I. Balabanovich and B. Schartel, Polymer, 2007, 48, 778790. 